OPEN ACCESS

Edited by:

Yuling Jiao,

Chinese Academy of Sciences, China

Reviewed by:

Honghui Lin,

Sichuan University, China

Mingyi Bai,

Shandong University, China

*Correspondence:

Hua Xu

xuhua@qibebt.ac.cn

Gongke Zhou

Zhougk@qau.edu.cn

Specialty section:

This article was submitted to

Plant Development and EvoDevo,

a section of the journal

Frontiers in Plant Science

Received: 22 July 2020

Accepted: 29 September 2020

Published: 27 October 2020

Citation:

Tang X, Wang C, LiU Y, He G, Ma N, Chai G, Li S, Xu H and Zhou G

(2020) Brassinosteroid Signaling

Converges With Auxin-Mediated C3H17 to Regulate Xylem Formation

in Populus.

Front. Plant Sci. 11:586014 doi: 10.3389/fpls.2020.586014

\section{Brassinosteroid Signaling Converges With Auxin-Mediated C3H17 to Regulate Xylem Formation in Populus}

\author{
Xianfeng Tang ${ }^{1}$, Congpeng Wang ${ }^{2}$, Yu Liu ${ }^{2}$, Guo He ${ }^{1}$, Nana Ma ${ }^{3}$, Guohua Chai ${ }^{2}$, \\ Shengjun $\mathrm{Li}^{1}$, Hua $\mathrm{Xu}^{1 *}$ and Gongke Zhou ${ }^{1,2 *}$
}

${ }^{1}$ Key Laboratory of Biofuels, Shandong Provincial Key Laboratory of Energy Genetics, Shandong Institute of Energy Technology, Qingdao Institute of Bioenergy and Bioprocess Technology, Chinese Academy of Sciences, Qingdao, China, ${ }^{2}$ College of Resources and Environment, Qingdao Agricultural University, Qingdao, China, ${ }^{3}$ State Key Laboratory of Crop Biology, College of Life Science, Shandong Agricultural University, Tai'an, China

Brassinosteroid (BR) signaling has long been reported to have an effect on xylem development, but the detailed mechanism remains unclear, especially in tree species. In this study, we find $\mathrm{PdC} 3 \mathrm{H} 17$, which was demonstrated to mediate xylem formation driven by auxin in our previous report, is also involved in BR-promoted xylem development. $\mathrm{Y} 1 \mathrm{H}$ analysis, EMSA, and transcription activation assay confirmed that $\mathrm{PdC} 3 \mathrm{H} 17$ was directly targeted by PdBES1, which is a key transcriptional regulator in BR signaling. Tissue specificity expression analysis and in situ assay revealed that PdC3H17 had an overlapping expression profile with PdBES1. Hormone treatment examinations verified that xylem phenotypes in $\mathrm{PdC} 3 \mathrm{H} 17$ transgenic plants, which were readily apparent in normal condition, were attenuated by treatment with either brassinolide or the BR biosynthesis inhibitor propiconazole. The subsequent quantitative real-time polymerase chain reaction ( $\mathrm{RRT}-\mathrm{PCR}$ ) analyses further revealed that BR converged with $\mathrm{PdC} 3 \mathrm{H} 17$ to influence transcription of downstream xylem-related genes. Additionally, the enhancement of xylem differentiation by auxin in $\mathrm{PdC} 3 \mathrm{H} 17$ overexpression plants was significantly attenuated compared with wild-type and dominant negative plants due to BR deficiency, which suggested that the BR- and auxin-responsive gene $\mathrm{PdC} 3 \mathrm{H} 17$ acted as an mediation of these two hormones to facilitate xylem development. Taken together, our results demonstrate that BR signaling converges with auxin-mediated $\mathrm{PdC} 3 \mathrm{H} 17$ to regulate xylem formation in Populus and thus provide insight into the regulation mechanism of BRs and the crosstalk with auxin signaling on xylem formation.

Keywords: populus, auxin, PdC3H17, brassinosteroids, xylem formation

\section{INTRODUCTION}

Xylem is a highly specialized vascular tissue with the roles of transporting water and minerals and also providing mechanical support for upright growth. The formation of xylem is mainly dependent on the activity of (pro)cambium and the rate of differentiation from it (Jouannet et al., 2015; Kondo et al., 2015). Several hormonal signals have been shown to be involved in maintenance of cambium 
and the formation of secondary vascular tissues, such as auxin, cytokinin, and gibberellin (Ragni et al., 2011; Ruzicka et al., 2015; Schaller et al., 2015). These hormones have been suggested to have different internal concentration gradients across woodforming tissues, and these gradients are highly related with the gene expression and different developmental stages during the formation of secondary vascular tissues (Bhalerao and Fischer, 2014; Immanen et al., 2016).

Brassinosteroids (BRs) are also demonstrated to be involved in vascular differentiation (Yamamoto et al., 1997; Kang et al., 2017). Several studies in herbaceous plants have shown that BRs were implicated in the differentiation of primary vascular cell types. During the in vitro differentiation of tracheary elements (TEs) in the Zinnia cell cultures system, exogenous application of brassinolide (BL) could promote the formation of TEs by inducing the expression of genes related with secondary cell wall (SCW) deposition and programmed cell death (PCD; Yamamoto et al., 1997). Consistent with the idea that BRs were required for the later stages of TE differentiation, five different types of BRs accumulated both within the cells and in the TE culture medium during TE differentiation in Zinnia (Yamamoto et al., 2001). Moreover, the levels of BR intermediates have been revealed to peak at the transition from undifferentiated cells to TE (Yamamoto et al., 2001). In Arabidopsis, two BR receptor members BRL1 and BRL3, which were dominantly expressed in vascular tissues, could promote xylem differentiation and repress phloem cell differentiation (Cano-Delgado et al., 2004). As a key component in BR signaling, BES1 was revealed to function as a downstream target of GSK3s during xylem differentiation in a TDIF-dependent manner (Kondo et al., 2014), which also indicated that BES1 was a shared common signaling component between TDIF signaling pathway and the BR signaling pathway in xylem development (Ryu et al., 2010; Guo et al., 2013; Kondo et al., 2014). Another similar result was obtained using VISUAL (Vascular cell Induction culture System Using Arabidopsis Leaves) system in Arabidopsis, indicating that BES1 and its homolog BZR1 redundantly promoted both phloem and xylem differentiation (Saito et al., 2018). Notably, BES1 had a stronger impact on vascular cell differentiation than BZR1, suggesting that BES1 had a key role in BR signaling during vascular differentiation (Saito et al., 2018). However, the mechanism of BES1 in vascular development, especially its downstream components, remains largely unknown. Recent studies also revealed that BR signaling was closely related to secondary xylem formation in trees. A reduced lignification and altered cell-wall carbohydrate biosynthesis occurred within secondary xylem of Liriodendron tulipifera trees through the exogenous application of BR (Jin et al., 2014). In poplar, BR could induce the expression of $B E E 3$, which encoded a basic helix-loop-helix transcription factor BR enhanced expression 3, resulting in enhanced secondary xylem formation (Noh et al., 2015). In addition, overexpression of several BR biosynthesis genes, such as CYP85A3, DWF4, and DET2, all showed increased secondary xylem formation in poplar due to an elevated level of BRs in vivo (Jin et al., 2017; Shen et al., 2018; Du et al., 2020; Fan et al., 2020). Recently, a key BR biosynthesis gene, PtiCYP85A3, was reported to play a critical role in BR-mediated tension wood formation in poplar (Jin et al., 2020). These studies suggest that BR signaling plays an important role in secondary growth and wood formation.

In addition, BRs also interplay with other hormones such as auxin during vascular differentiation (Hardtke, 2007; Sorce et al., 2013). In primary growth, BRs act in concert with auxin during the patterning of vascular bundles in the stem (Reinhardt et al., 2003; Sauer et al., 2006). BRs were predicted to control the bundle number by promoting the early cell divisions of procambial. In contrast, auxin maxima had a crucial role in defining spacing of the vascular bundle (Ibanes et al., 2009). But so far, the key components especially transcriptional factors (TFs) involved in BR signaling and the linkage with other hormones during secondary xylem formation are far from revealed.

The $\mathrm{CCCH}$ proteins possess a $\mathrm{CCCH}$ zinc finger motif composed of three cysteines followed by one histidine and have been found widely in eukaryotes (Fu and Blackshear, 2017). This type of proteins is conventionally known to be involved in functioning at both the transcriptional and posttranscriptional levels in animals (Lai et al., 2000; Lai and Blackshear, 2001; Hudson et al., 2004). Functional studies have revealed that plant $\mathrm{CCCH}$ zinc finger proteins play key roles in plant growth, hormone responses, and biotic/abiotic stress responses (Kim et al., 2008; Seok et al., 2016; Zhang et al., 2019). Our recent work revealed that $\mathrm{CCCH}$ protein $\mathrm{PdC} 3 \mathrm{H} 17$ could form a module with its partner PdMYB199 driven by auxin in xylem formation (Tang et al., 2020). However, the connections between CCCH TFs and BRs during xylem formation have not yet been studied. It is noteworthy that such a connection is likely to exist, as RNA-seq data indicated that some cell differentiation and SCW biosynthesis genes were not only influenced by BR signaling, but also regulated by PdC3H17 (Du et al., 2020; Tang et al., 2020).

In this study, we tested the hypothesis that $\mathrm{PdC} 3 \mathrm{H} 17$ interacted with BR signaling pathway in the modulation of xylem formation. Our results indicated that the function of PdC3H17 on xylem cell differentiation was mediated by BR signaling. Biochemical and molecular analysis indicated that PdC3H17 acted as a downstream TF of PdBES1. The exogenous propiconazole $(\mathrm{PCZ})$ treatment revealed that $\mathrm{PdC} 3 \mathrm{H} 17$ was a positive regulator in xylem formation since the $P d C 3 H 17 D R$ plants (dominant repression) displayed lower sensitivity to BR. Further, inhibition of BR synthesis reduced auxin sensitivity differences on xylogenesis between PdC3H17DR and overexpression plants, suggesting that $\mathrm{PdC} 3 \mathrm{H} 17$ might be a co-effector linking auxin and BR signaling during secondary xylem formation.

\section{MATERIALS AND METHODS}

\section{Plant Materials and Growth Conditions}

Populus "Nanlin 895" was used in this study and as the background of $\mathrm{PdC} 3 \mathrm{H} 17$ dominant negative and overexpression transformation. The 35S:FLAG-PdC3H17 (PdC3H17OE) and $P d C 3 H 17-D R$ transgenic plants used in this study were described in our previous reports (Chai et al., 2014a; Tang et al., 2020) 
and were grown in sterilized tissue culture bottle at $22^{\circ} \mathrm{C}$ under long-day conditions (16-h light/8-h dark).

Arabidopsis thaliana ecotype Columbia (Col-0) was used in this study for analysis of protoplast transcription activation activity, which were grown in soil at $22^{\circ} \mathrm{C}$ under long-day conditions (16-h light/8-h dark).

\section{Hormone Treatment Analyses}

Four-week-old wild-type (WT) Populus "Nanlin 895" were incubated with the hormone solutions. The concentrations for the treatments were 0 (control), 1, 10, and $50 \mu \mathrm{M} \mathrm{BL}$ (Epibrassinolide; Sigma-Aldrich, St. Louis, MO, United States). BL-treated stems were subjected to $\mathrm{PdC} 3 \mathrm{H} 17$ expression analysis by quantitative real-time polymerase chain reaction (qRT-PCR). Three biological replicates were performed independently.

To analyze the responsiveness of BRs or auxin with BRs on xylem formation, at least three independent lines of rootremoved control and transgenic seedlings were grown on the 1/2 MS medium with or without $\mathrm{PCZ} /(\mathrm{PCZ}+\mathrm{IBA})$ at various determined concentrations for 21 days ( $8 \mathrm{~h}$ for gene expression analysis), and $0.3-\mathrm{cm}$ segments were taken from their basal stems. These segments were fixed in $4 \%$ paraformaldehyde (Sigma-Aldrich) at $4^{\circ} \mathrm{C}$ for 4 days, dehydrated in graded ethanol series, and embedded into paraplast. The $5-\mu \mathrm{m}$ sections were obtained using a Leica RM2235 rotary microtome and adhered to Superfrost microscope slides (Thermo). The sections were stained with $0.1 \%$ toluidine blue and observed using an Olympus X51 light microscope (Olympus). Radial widths of xylem were measured in three independent replicates using the SmileView software (JEOL).

\section{Real-Time Quantitative PCR}

Total RNA was extracted from hormone-treated poplar stems or various tissues of 4-month-old WT Populus "Nanlin 895," and first-strand cDNA syntheses were carried out as described previously (Li et al., 2018). qRT-PCR assays were performed on a LightCycler ${ }^{\circledR} 480$ Detection System (Roche) with TransStart Green qPCR superMix (TransGen Biotech). PdUBQ10 was used as an internal control, and data represent the average of three biological replicates. Primers of the related genes are listed in Supplementary Table S1.

\section{Transient Gene Expression Assays}

Arabidopsis leaf protoplasts transient assays were carried out following the procedure described previously (Tang et al., 2015). A 500-bp PdC3H17 promoter sequence (including E-box mutation) was ligated upstream of the $\beta$-glucuronidase (GUS) reporter after removing the $35 \mathrm{~S}$ promoter in $\mathrm{pBI} 221$ vector to create the reporter constructs. The PdBES1 coding region was ligated between the $35 \mathrm{~S}$ promoter and the NOS terminator after removing GUS from the pBI221 vector to create the effector construct. The reporter and effector constructs as well as the 35S:LUC vector (internal control) were co-transformed into Arabidopsis leaf protoplasts. Samples were incubated overnight in darkness and then conducted the GUS and luciferase enzymatic assays. The reporter gene expression levels were determined as the relative ratio of GUS to luciferase activity. Data were the means of three biological replications.

\section{In situ Analysis}

The 163-, 125-, and 143-bp CDS regions of PdC3H17, PdBES1 (Potri.014G041600), and PdBZR1 (Potri:011G106800) were separately used to synthesize the digoxigenin-labeled antisense and sense RNA probes with DIG RNA Labeling mix (Roche). The specific primers are listed in Supplementary Table S1. The 10th internode of 1.5-m-high Populus "Nanlin 895" plants growing in a glasshouse was sectioned, fixed in $4 \%$ glutaraldehyde, and embedded in paraffin. Stem section $(8 \mu \mathrm{m})$ was cut on a rotary Leica RM 6025 microtome (Leica), mounted onto Superfrost glass slides (Thermo Fisher), and hybridized with the antisense or sense RNA probes. Hybridization and immunological detection were carried out according to the previous description (Xu C. et al., 2019).

\section{Yeast One-Hybrid Assay}

The yeast one-hybrid assay was conducted as described previously (Wang et al., 2020). The coding sequence of PdBES1 was inserted into pGADT7 vector to form pGADT7-BES1. The three tandem repeats of the E-box (CANNTG) motif in PdC3H17 promoter were combined to pHIS2.1 to form bait reporter vector. Then, pGADT7-BES1 and pHIS-E-box (pHIS-Ev as negative control) vectors were transferred to yeast-one-hybrid yeast strain, followed by culture on SD/-Leu/-Trp medium for 3 days. Positive clones were then spread on SD/-Leu/-Trp and $\mathrm{SD} /$-Leu/-Trp/-His (15 mM 3AT) medium, respectively.

\section{EMSA Analysis}

The PdBES1 coding region was fused in frame with MBP in the pMAL-p4x vector. Recombinant protein was expressed and purified from Escherichia coli using amylose resin (NEB). Biotin-labeled synthetic oligonucleotide was annealed with unlabeled oligonucleotides and then used as probes. A DNA protein-binding reaction was performed using a LightShift ${ }^{\circledR}$ Chemiluminescent EMSA Kit (Pierce) according to the previous description (Xu Z. et al., 2019). Briefly, the labeled DNA fragments were incubated for $25 \mathrm{~min}$ with $180 \mathrm{ng}$ of the recombinant protein in binding buffer $[10 \mathrm{mM}$ Tris $(\mathrm{pH}$ 7.5), $1 \mathrm{mM}$ DTT, 2.5\% glycerol, $50 \mathrm{mM}$ KCL, $10 \mathrm{mM}$ $\mathrm{MgCl}_{2}, 0.1 \%$ Nonidet $\mathrm{P}-40$, and $50 \mathrm{ng} / \mu \mathrm{L}$ poly (dI-dC)]. The protein-DNA fragments were separated from the unbound fragments by polyacrylamide gel electrophoresis. The DNA was electroblotted onto a nitrocellulose membrane and detected by chemiluminescence. The experiments were performed for three independent times.

\section{RESULTS}

\section{PdC3H17 Is a Direct Target of PdBES1}

Previous study has demonstrated that $\mathrm{PdC} 3 \mathrm{H} 17$ was involved in xylem formation by interacting with auxin signaling (Tang et al., 2020). To identify the putative upstream components that regulate $\mathrm{PdC} 3 \mathrm{H} 17$, a yeast one-hybrid $(\mathrm{Y} 1 \mathrm{H})$ screen assay was 
performed using poplar xylem-specific cDNA library. As bait, a 102-bp $\mathrm{PdC} 3 \mathrm{H} 17$ promoter segment including various potential binding sites analyzed in NCBI was fused to pHIS2.1 vector. A total of 18 xylem-expressed genes were found to possibly bind to the $\mathrm{PdC} 3 \mathrm{H} 17$ promoter sequence (Supplementary Table S2). Among these candidates, Potri.014G041600 represented the most promising one since its homolog in Arabidopsis was AtBES1, which plays a central role in the regulation of xylem formation in BR signaling. We thus named Potri.014G041600 as PdBES1. In order to determine whether PdC3H17 could be a target of PdBES1, we first conducted $\mathrm{PdC} 3 \mathrm{H} 17$ promoter sequence analysis; the result showed that multiple E-box elements (CANNTG), which are putative BES1 binding site, were found within $-500 \mathrm{bp}$ relative to the transcription start site (Supplementary Figure S1). We then performed yeast onehybrid assays and detection of strong reporter gene activation in yeast co-expressing AD-PdBES1 and PHis2.1-C3H17pro in $\mathrm{Y} 1 \mathrm{H}$ system, which indicated that PdBES1 may have a connection with
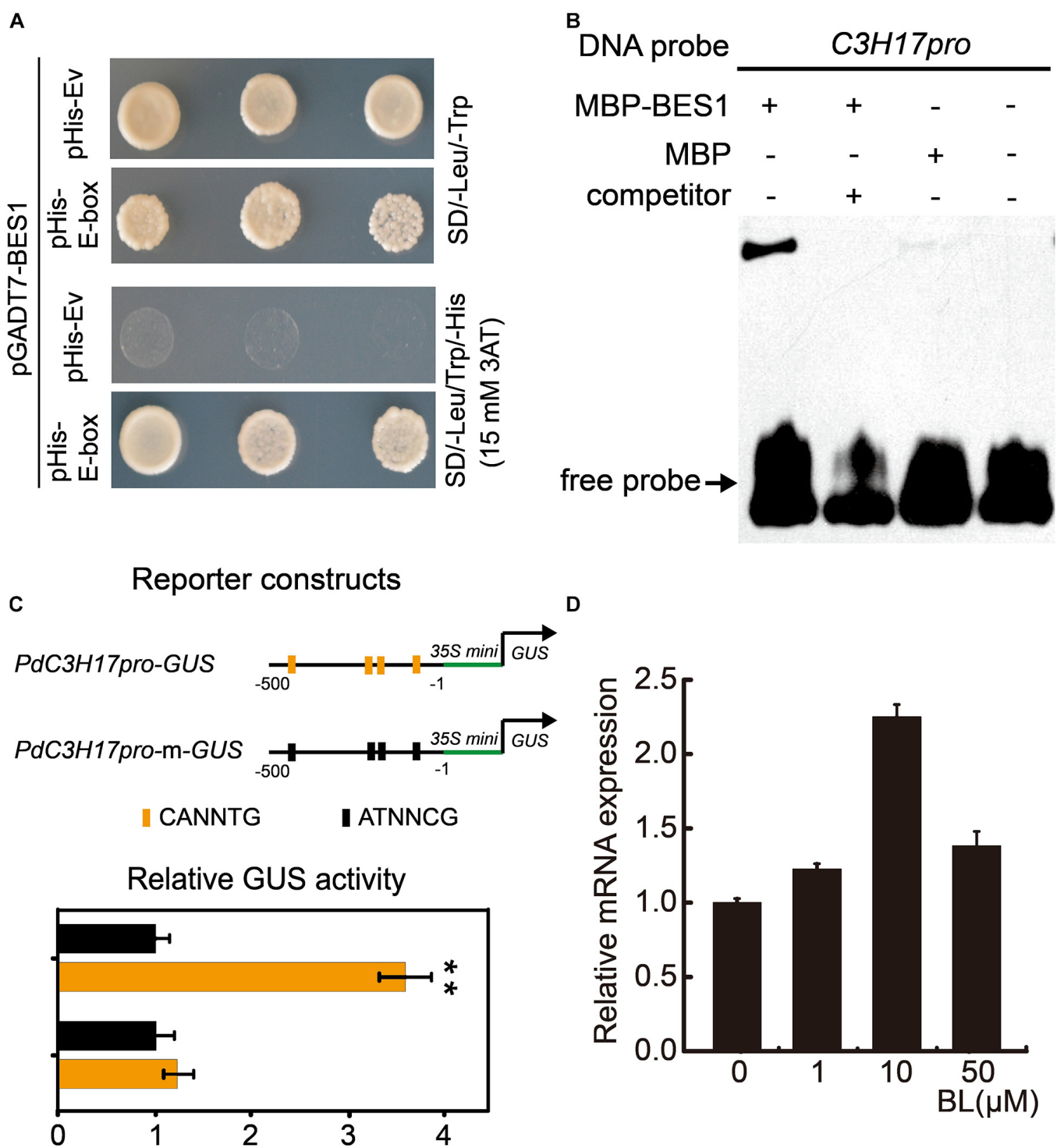

D

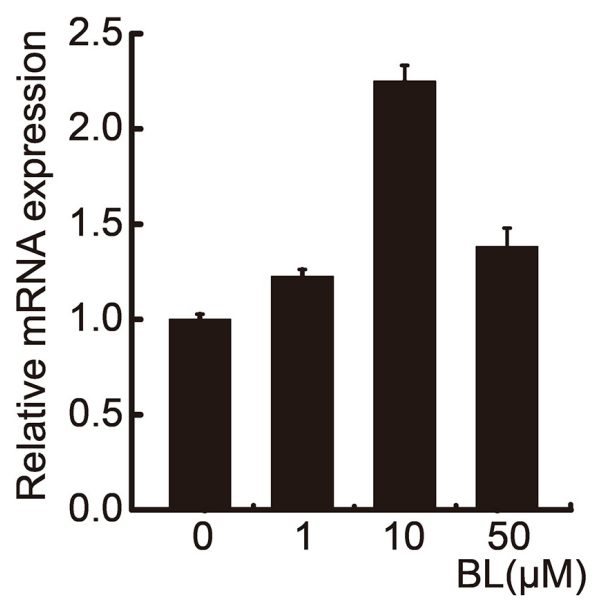

FIGURE 1 | PdBES1 directly activates PdC3H17 in vitro. (A) Y1H analysis of PdBES1 binding to the PdC3H17 promoter sequence indicated in Supplementary Figure S1. pHis-E-box showed recombinant $\mathrm{pHis}$ vector contained E-box (CANNTG) motif in PdC3H17 promoter, pHis-Ev represented pHis empty vector. (B) EMSA analysis showed the specific binding of PdBES1 protein to the promoter fragment of $\mathrm{PdC} 3 \mathrm{H} 17$ in vitro. (C) Transcription activity assay showed that PdBES1 activated the PdC3H17 promoter-driven expression of the GUS reporter gene. For reporters, a short 500-bp promoter fragment of $P d C 3 H 17$ was constructed to drive the expression of GUS. The E-box harbored in these promoter fragments was disrupted via site-directed mutagenesis for generating PdC3H17pro-m-GUS. The effector encodes PdBES1 driven by the CaMV 35S promoter. The relative ratio of GUS to luciferase activity after cotransformation into Arabidopsis protoplast cells with different reporter and effector construct combinations was tested. Values for blank effector were normalized to 1 . Error bars represent SD. Student $t$-test was performed to evaluate significant difference between values of blank effector and those of PdBES1 $\left(t\right.$-test: $\left.{ }^{\star \star} P<0.01, n=3\right)$. (D) Transcript levels of $\mathrm{PdC} 3 \mathrm{H} 17$ are shown after application of various concentrations of 24-eBL for $4 \mathrm{~h}$. PdUBQ10 was used as an internal control. 
these binding sites located in $\mathrm{PdC} 3 \mathrm{H} 17$ promoter (Figure 1A). To further confirm this observation, we then purified PdBES1 protein fused with MBP tag from E. coli and conducted EMSA analysis in vitro. As shown in Figure 1B, PdBES1 specifically bound to $\mathrm{PdC} 3 \mathrm{H} 17$ promoter fragment (Figure 1B). To validate direct regulation of PdBES1 on $\mathrm{PdC} 3 \mathrm{H} 17$ via binding to these E-box elements, short promoter fragments harboring these E-box elements were constructed to drive the GUS reporter for transcriptional activation assays. Consistently, PdBES1 significantly activated the expression of GUS reporter driven by the E-box-harbored promoter fragments of $\mathrm{PdC} 3 \mathrm{H} 17$. By contrast, the PdBES1-driven activation disappeared when the E-box elements harbored in the promoter fragments of $\mathrm{PdC} 3 \mathrm{H} 17$ were disrupted by site-directed mutagenesis (Figure 1C). The direct regulation of PdBES1 on $\mathrm{PdC} 3 \mathrm{H} 17$ prompted us to ask whether there is a connection between PdC3H17 and BR signaling. We then examined the expression profiles of $\mathrm{PdC} 3 \mathrm{H} 17$ in poplar plantlets (grown for 4 weeks in vitro) at various concentrations of $\mathrm{BL}$ (the most active BR). Total RNA was extracted from the BL-treated stems and subjected to the qRTPCR using PdC3H17-specific primers. The results showed that the transcript level of $\mathrm{PdC} 3 \mathrm{H} 17$ was almost not changed after $1 \mu \mathrm{M}$ BL treatment but was induced approximately twofold after $10 \mu \mathrm{M}$ BL treatment. However, its expression was decreased again after $50 \mu \mathrm{M}$ BL treatment (Figure 1D). These results indicated that PdBES1 could directly target $\mathrm{PdC} 3 \mathrm{H} 17$ and induce its expression in BR signaling pathway.

Since $\mathrm{PdC} 3 \mathrm{H} 17$ could be a target of PdBES1 in vitro and then whether there is a connection between these two genes in vivo during xylem formation. We explored it through detecting their expression profiles in wood-forming tissues because the function of PdBES1 in xylem formation has not yet been studied. Except for PdBES1, as comparison, we also analyzed the expression patterns of PdBZR1 (Potri.011G106800), homologous gene of $P d B E S 1$ in BR signaling pathway. Tissuespecific expression analysis showed that PdBZR1 and PdBES1 were expressed in all tested tissues, which were consistent with their homologies in Arabidopsis (Yin et al., 2002; He et al., 2005). Besides, in accordance with the expression pattern of $P d C 3 H 17, P d B Z R 1 / B E S 1$ were highly expressed in the middle and basal portion of stems, where the cells are undergoing secondary wall thickening and xylem formation (Figure 2A). Further, the continuous assays across secondary xylem tissues in poplar stems allowed us to evaluate spatial expression patterns of these highly wood tissue expressed genes (Sundell et al., 2017). By using this database, a highly similar expression pattern of $\mathrm{PdC} 3 \mathrm{H} 17$ and $P d B E S 1$ was obtained, with two expression peaks in cambium zone and differentiated xylem zone. By contrast, low similarity across the wood-forming tissues was revealed between $\mathrm{PdC} 3 \mathrm{H} 17$ and PdBZR1 (Figure 2B), which suggested a potential connection between $P d C 3 H 17$ and $P d B E S 1$ during xylem formation.

To obtain detailed expression patterns of PdBZR1/BES1 in secondary vascular tissues, RNA in situ hybridization was performed using the fourth internode of 1.5-month-old poplar (Figure 2C). The transcripts of PdBES1 were preferentially accumulated in cambial zone and closely neighboring cell layers of the wood-developing stem, which was almost identical with the observation of distribution of $\mathrm{PdC} 3 \mathrm{H} 17$ across the stem with predominance expression in cambium adjacent cells toward xylem differentiation (Figure 2C). However, PdBZR1 was distributed only in developing xylem, which was consistent with the analysis from Sundell and colleagues' data. Taken together, the similar expression patterns indicated that $\mathrm{PdC} 3 \mathrm{H} 17$ could be involved in BR signaling pathway downstream of PdBES1 during xylem formation.

\section{PdC3H17 Transgenic Plants Show Altered Response to BR During Xylem Formation}

To evaluate the effect of BRs on the xylem formation in $P d C 3 H 17$ transgenic plants, a dose-response curve was generated for control (Nanlin 895), PdC3H17OE, and PdC3H17DR plants grown in the presence of various concentrations of exogenous PCZ (a BR synthesis inhibitor), which generally inhibits secondary xylem formation in stems (Du et al., 2020). The results showed that the xylem formation was repressed in all three genotypes when treated with PCZ, which was in agreement with the previous study (Du et al., 2020), but the transgenic plants exhibited different responses to PCZ as compared with the control. The $P d C 3 H 17 D R$ plants had smaller reduced xylem formation compared to the control. More significantly, the $P d C 3 H 17 D R$ plants produced almost the same xylem as in the control ones in the presence of $3 \mu \mathrm{M}$ PCZ, whereas the xylem formation in overexpression plants decreased more drastically compared with the control ones (Figure 3). The results indicated that $P d C 3 H 17 D R$ plants were less sensitive to inhibition of xylem formation caused by PCZ treatment, whereas PdC3H17OE plants were more sensitive (Figure $\mathbf{3 K}$ ). This suggested that BRs were important for modulation of xylem formation by $\mathrm{PdC} 3 \mathrm{H} 17$. To further test this hypothesis, we also generated dose-response curves for all three genotypes grown in the presence of BL. As expected, BL treatment had the opposite effect as compared with PCZ (Figure 4). Xylem formation was promoted by BL, and the promotion effect was more significant in $P d C 3 H 17 D R$ as compared with the WT. However, the effect of BL was less significant in $\mathrm{PdC} 3 \mathrm{H} 17 \mathrm{OE}$ plants (Figure $4 \mathrm{~K}$ ). These results further suggested that there was a connection between PdC3H17 function and BR signaling during xylem formation.

\section{BR Signaling Regulates a Subset of PdC3H17-Mediated Xylem-Related Genes}

Our previous study revealed that $\mathrm{PdC} 3 \mathrm{H} 17$ affected xylem formation through modulation of SCW deposition and PCD (Chai et al., 2014a; Tang et al., 2020). One possible explanation for the results observed in the xylem formation assays may be that $\mathrm{BR}$ signaling affected $\mathrm{PdC} 3 \mathrm{H} 17$ regulation of downstream xylem-related genes. To test this, gene expression was examined in seedlings grown in the presence or absence of PCZ using qRTPCR. The expression of these xylem-related genes was decreased in both control and $P d C 3 H 17 O E$ plants grown in the presence of PCZ compared with the MeoH mock, although this decrease was not statistically significant in control plants (Figures 5A,B). In contrast, no decrease of this gene expression was observed in $P d C 3 H 17 D R$ plants grown in the presence of PCZ (Figure 5C). 


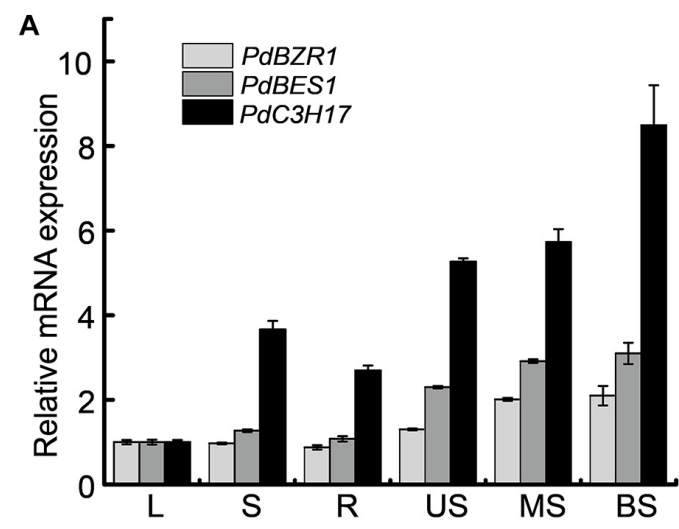

C

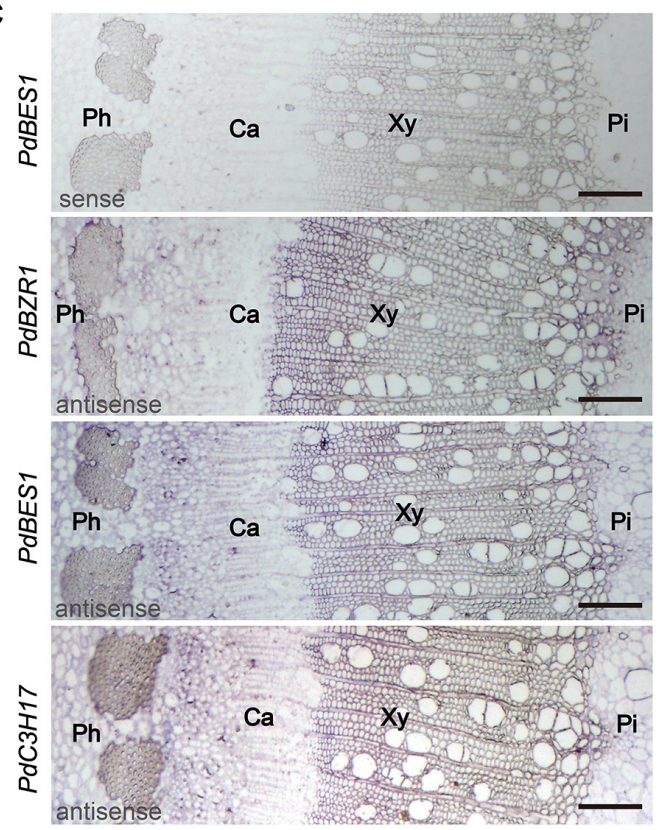

B
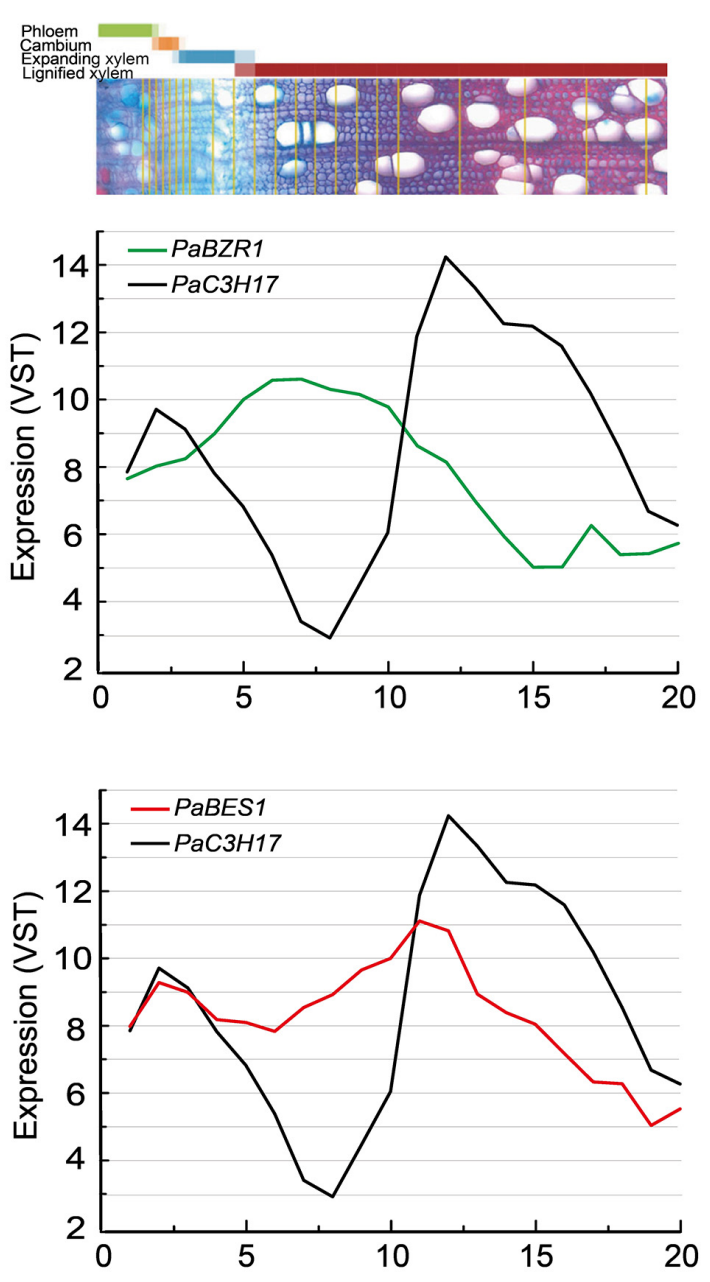

FIGURE 2 | The comparison of expression patterns of PdBZR1, PdBES1, and PdC3H17. (A) The transcript levels of PdBZR1, PdBES1, and PdC3H17 were measured in various poplar tissues. L: leaf; S: shoot; R: root; US: upper stem; MS: middle stem; BS: basal stem. Data are means \pm SD of three biological repeats. (B) Expression patterns of PdBZR1, PdBES1, and PdC3H17 genes across secondary stem tissues based on the AspWood RNAseq database (Sundell et al., 2017). The curves were generated using the values for the cryosection of stem tissues. The number in $x$ axis showed continuous cryosection from phloem to lignified xylem. (C) In situ hybridization assay of PdBZR1, PdBES1, and PdC3H17 in poplar stem. Cross-sections of the basal stems were hybridized with digoxigenin-labeled antisense RNA probes of PdBZR1, PdBES1, and PdC3H17 or with a digoxigenin-labeled sense PdBES1 RNA probe as a control. The hybridization signals are shown in purple. Ph: phloem; Ca: cambium; Xy: xylem; Pi: pith. Bars: $100 \mu \mathrm{m}$.

A battery of poplar MYB transcription factors that are involved in the transcriptional network mediated by MYB3/21 has previously been shown to play a key role in secondary xylem formation (Zhong et al., 2011), and these TFs were demonstrated to be regulated in $P d C 3 H 17 O E$ and $P d C 3 H 17 D R$ plants (Chai et al., 2014a). Therefore, we investigated whether these TFs transcripts are affected by inhibition of BR synthesis. The qRT-PCR results showed that of all six TFs, only the expression of PdMYB121, $P d M Y B 125$, and PdMYB167 changed significantly after PCZ treatment, the PCZ treatment reduced the differences of these gene expression level among all three genotypes. However, expression levels of other genes had no obvious change in transgenic or control plants after PCZ treatment, suggesting that a unique transcriptional mechanism mediated by BR signaling might exist to impact xylem formation in $\mathrm{PdC} 3 \mathrm{H} 17$ transgenic lines (Supplementary Figure S2). These results indicated that BR signaling functioned to modulate the expression of xylem-related genes in $\mathrm{PdC} 3 \mathrm{H} 17$ transgenic plants.

We also examined several genes expression regulated by both $\mathrm{BR}$ and $\mathrm{PdC} 3 \mathrm{H} 17$ in $\mathrm{PdC} 3 \mathrm{H} 17 \mathrm{DR}$ plants, including $\mathrm{XTH33}, \mathrm{C} 4 \mathrm{H}$, XND1, and ERF1 (Du et al., 2020; Tang et al., 2020). In general, the expression levels of these genes were decreased either in the absence or presence of BL in the $P d C 3 H 17 D R$ plants compared to the control ones (Figure 5D). The BL induction was significantly decreased in at least three test genes $(X T H 33, C 4 H$, and XND1). Taken together, the gene expression studies suggested that BR 

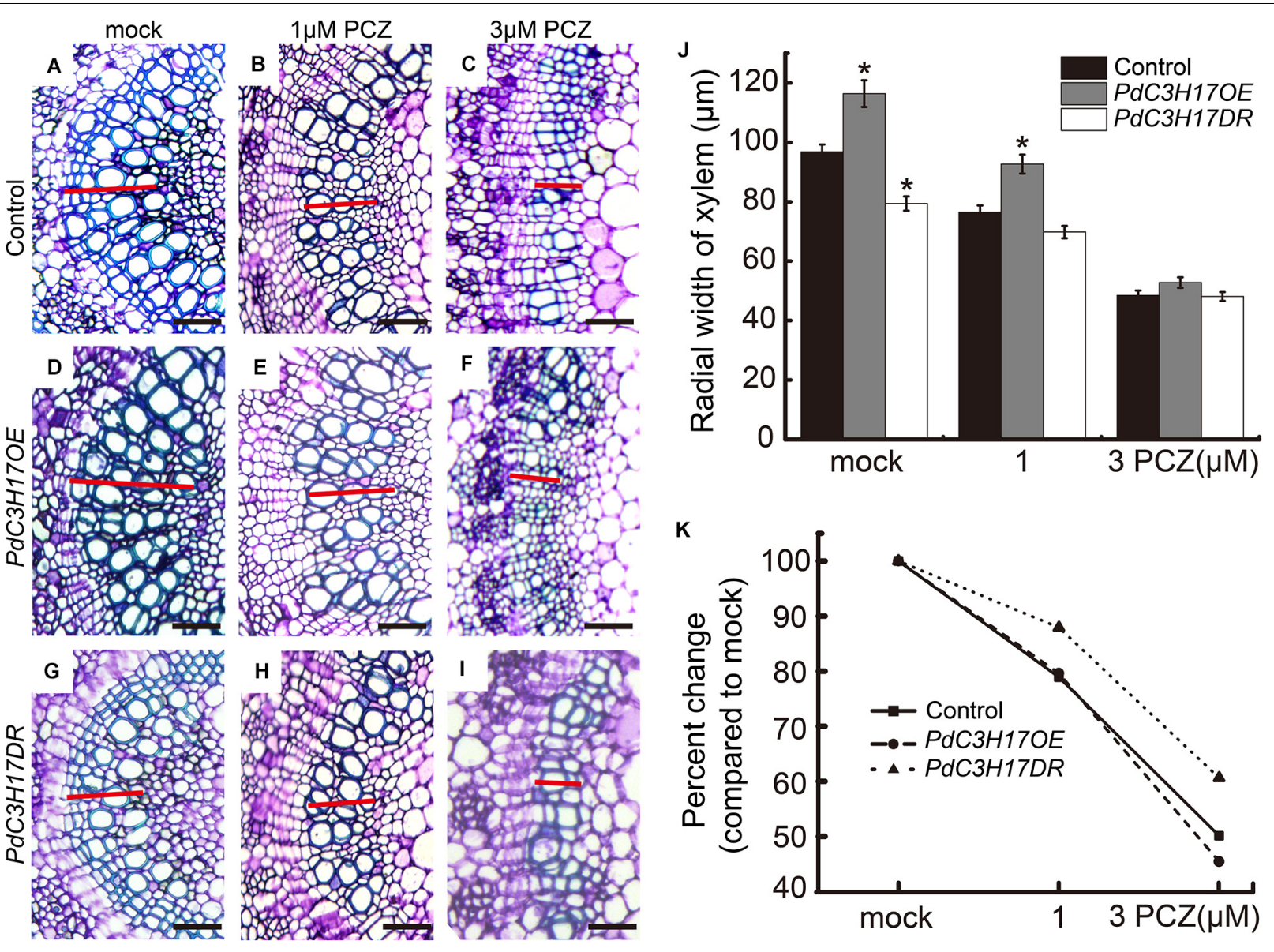

FIGURE 3 | PdC3H17 overexpression and dominant repressor plants show altered brassinosteroid (BR) response phenotypes during xylem formation. (A-I) BR responsiveness assays in the developing stems (xylem) of $\mathrm{PdC} 3 \mathrm{H} 17$ transgenic plants. Microscopic analysis of basal stem sections of the control (A-C), PdC3H17OE (D-F), and PdC3H17DR (G-I) plants grown in 1/2 MS medium containing 0,1 , and $3 \mu \mathrm{M}$ propiconazole (PCZ), respectively, for 21 days. The red lines indicate the width of xylem, bars: $50 \mu \mathrm{m}$. (J) Statistical analysis of the radial width of xylem in the stems of the control and PdC3H17 transgenic plants treated with PCZ in (A-I). (K) Percent changes in xylem width compared with plants of the same genotype grown with no PCZ treatment. For each construct, at least three independent lines were used for measurement of xylem radial widths, and three biological replicates were performed independently. $t$-test: ${ }^{*} P<0.05$.

signaling was essential for the optimal expression of a subset of PdC3H17 downstream components during xylem formation.

\section{BR Converges With Auxin Signaling to Impact Xylem Formation in $\mathrm{PdC} 3 \mathrm{H} 17$ Transgenic Plants}

$\mathrm{PdC} 3 \mathrm{H} 17$ was demonstrated to promote xylem formation at least in part by the control of auxin signaling pathway in our previous study (Tang et al., 2020). Multiple auxin and BR signaling components physically interact with each other and synergistically affect various physiological processes, such as cell elongation and division, vascular differentiation, and root development (Bao et al., 2004; Woodward and Bartel, 2005). Therefore, we hypothesized that the altered sensitivities of $P d C 3 H 17 D R$ and $P d C 3 H 17 O E$ plants to auxin during xylem formation might be related to C3H17's impact on BR signaling. In order to test this hypothesis, we investigated xylem formation phenotypes of $\mathrm{PdC} 3 \mathrm{H} 17$ transgenic seedlings grown in $1 / 2 \mathrm{MS}$ medium containing $2 \mu \mathrm{M}$ IBA (the active form of auxin) and various concentrations of PCZ. The results showed that IBA treatment could significantly promote xylem formation in $P d C 3 H 17 O E$ plant compared with control and $P d C 3 H 17 D R$ plants, which was consistent with the previous study (Tang et al., 2020). Interestingly, we found that PCZ effectively blocked the enhanced sensitivity of $P d C 3 H 17 O E$ plants to auxin (Figures $6 \mathbf{F}-\mathbf{J}$ ). Most notably, there was almost no difference in xylem formation between control and PdC3H17OE plants grown in the presence of $2 \mu \mathrm{M} \mathrm{IBA}$ and $3 \mu \mathrm{M}$ PCZ (Figures 6E,J). In addition, PCZ treatment attenuated the defect xylem phenotype of $P d C 3 H 17 D R$ plants observed in the presence of IBA. These results suggested that $\mathrm{BR}$ signaling was important for the auxin-associated xylem formation observed in the $\mathrm{PdC} 3 \mathrm{H} 17$ transgenic plants.

As one of the outputs of auxin and BR signaling interaction, some transcription factors activated by these two different signaling pathways bind to the promoters of many shared target 


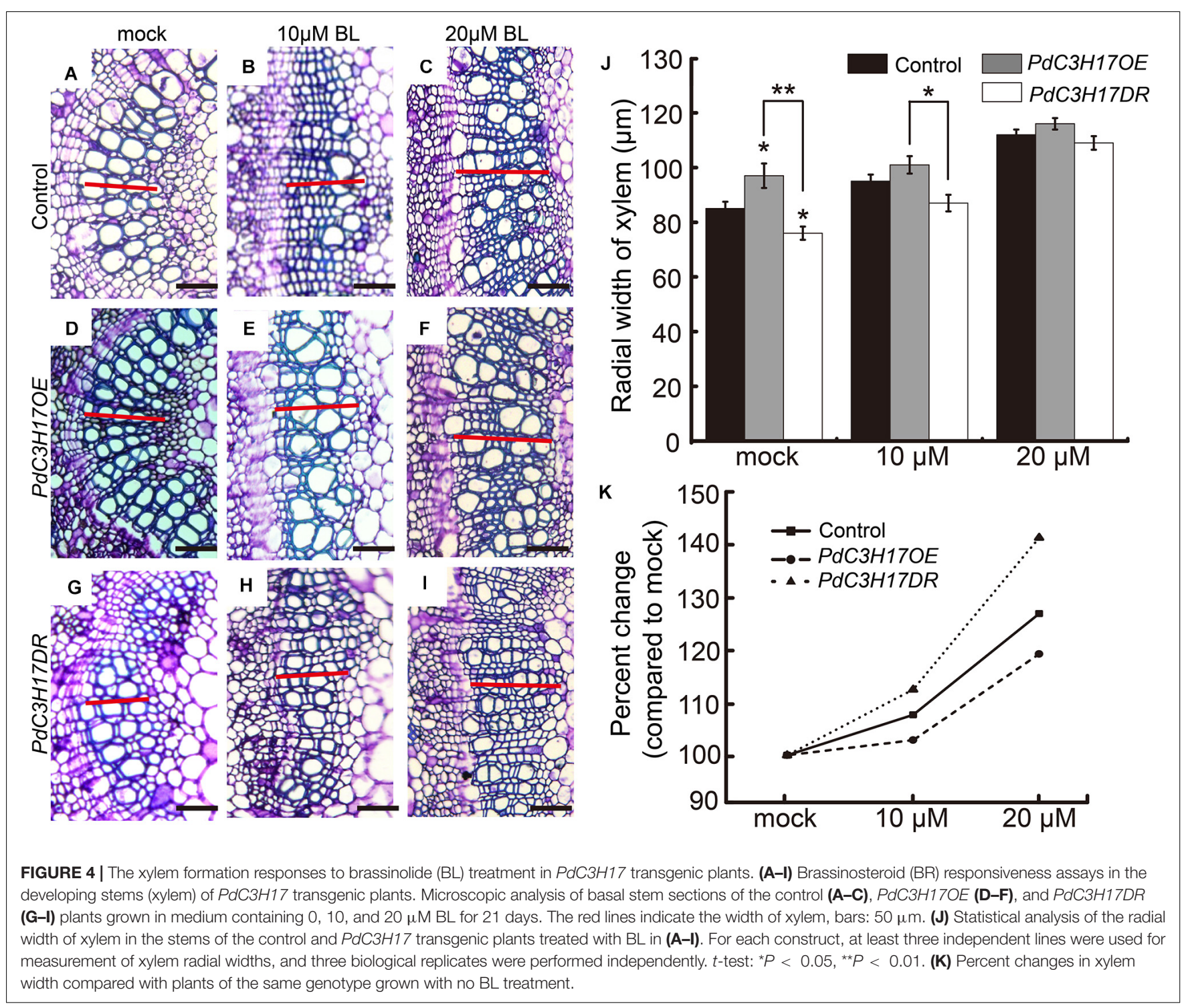

genes, synergistically regulating their transcription (Oh et al., 2014). In our previous study, we demonstrated that auxinmediated PdC3H17-MYB199 module could promote xylem formation through the regulation of expression of genes related with xylem formation (Tang et al., 2020). In the current study, the inhibition of BR could attenuate the increased xylem formation driven by auxin in the $\mathrm{PdC} 3 \mathrm{H} 17$ transgenic plants (Figure 6). To investigate whether BR affects the expression of $\mathrm{C} 3 \mathrm{H} 17$ 's target genes in this process, the transcripts of four genes in our previous study (two genes of CYCD3 and ERF109-1 associated with cell division, two genes of IRX10 and IRX15L-1 associated with SCW deposition) were detected. The qRT-PCR analysis revealed that auxin enhanced PdC3H17driven activation of the four genes' expression (Figure 7), which was in accordance with the previous study, whereas PCZ treatment attenuated the expression of these genes driven by auxin (Figure 7). These results suggested that BR interacted with auxin signaling in promoting xylem formation through a co-regulation of downstream xylem-related genes mediated by $\mathrm{PdC} 3 \mathrm{H} 17$.

\section{DISCUSSION}

Brassinosteroid has been recognized to be involved in xylem formation; many studies in Arabidopsis indicated that multiple components in BR signaling, including receptors and key TFs, have been revealed to differently regulate xylem formation (Cano-Delgado et al., 2004; Saito et al., 2018). Although we are starting to get a better insight into BR regulation of xylem formation in Arabidopsis, our understanding of the downstream regulation network of BR signaling during xylem formation in trees is limited. In this study, we characterized a $\mathrm{CCCH}$ protein $\mathrm{PdC} 3 \mathrm{H} 17$, which has been demonstrated to have a role in xylem formation (Chai et al., 2014a), to participate in this process through involving in BR signaling pathway in poplar. Indeed, 

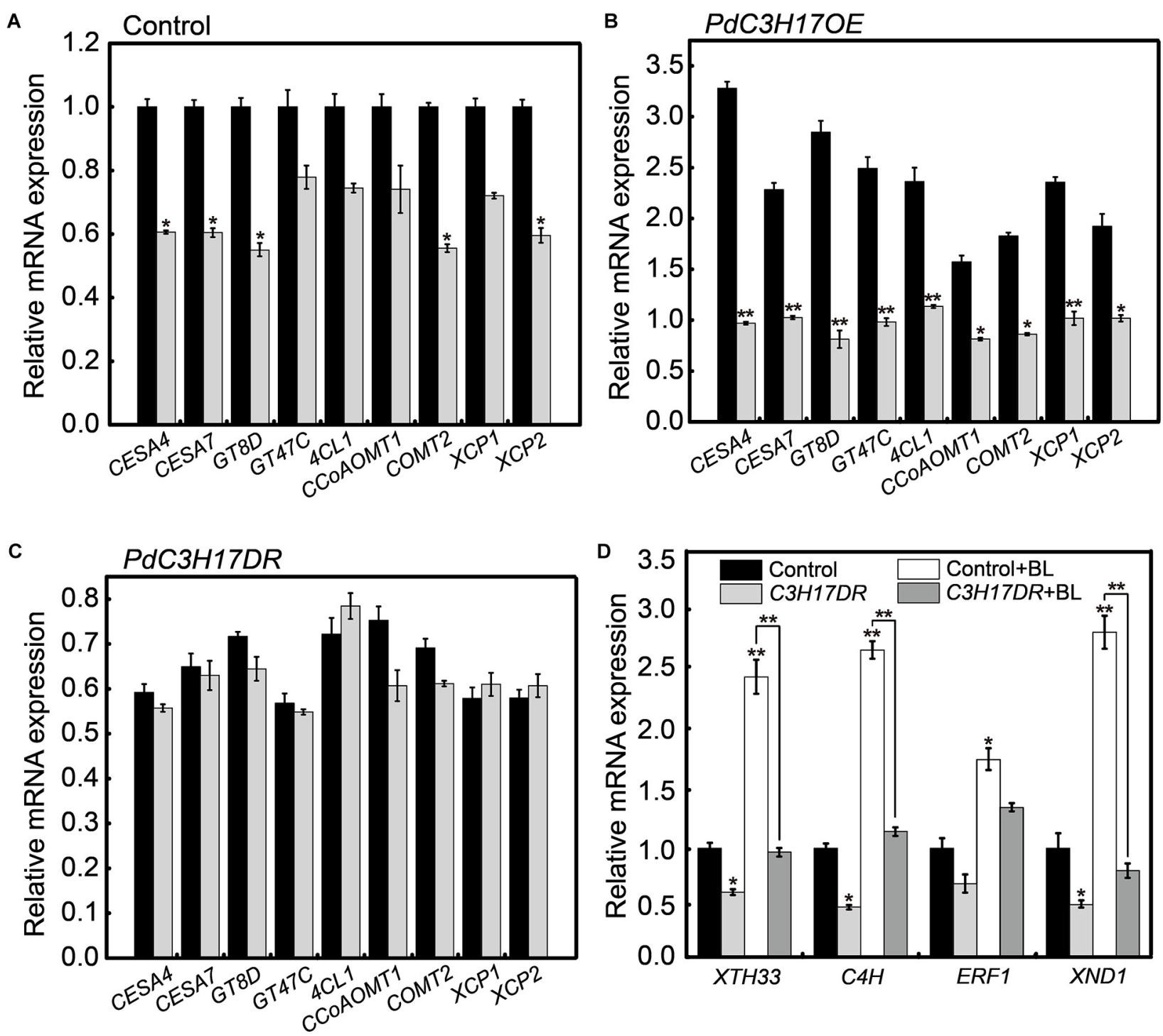

FIGURE 5 | Brassinosteroid (BR) affects the expression of a subset of PdC3H17-mediated xylem-related genes. (A-C) qRT-PCR analysis of several PdC3H17-mediated xylem-related gene expression in control (A), PdC3H17OE (B), and PdC3H17DR (C) plants treated with no (black column) and $3 \mu \mathrm{M}$ propiconazole (PCZ; gray column) for $8 \mathrm{~h}$. The expression values of these genes in control $\mathbf{( A )}$ with no PCZ treatment were set to 1 . $t$-test: ${ }^{\star} P<0.05$, ${ }^{\star \star} P<0.01$. (D) BR-induced gene expression is reduced in $P d C 3 H 17 D R$ plants with or without brassinolide (BL) treatment. Data are means \pm SD of three biological replicates. t-test: ${ }^{\star} P<0.05,{ }^{\star \star} P<0.01$.

PdC3H17 was markedly upregulated after BL treatment in poplar stem (Figure 1D), indicating that $\mathrm{PdC} 3 \mathrm{H} 17$ had a possible link with BR in xylem formation. The following hormone treatment examinations further verified that BR has an important impact on PdC3H17-mediated xylem formation. Although our gene expression analyses suggested that the physiological results of this study were at least partially explained by the convergence of $\mathrm{PdC} 3 \mathrm{H} 17$ and $\mathrm{BR}$ at the level of transcriptional regulation of genes encoding enzymes, which are directly involved in xylem formation, e.g., CESAs and $X$ (Figure 5), there are other potential explanations for the altered sensitivities of the xylem formation in $\mathrm{PdC} 3 \mathrm{H} 17$ transgenic plants to $\mathrm{BL}$ and PCZ. For example, $\mathrm{PdC} 3 \mathrm{H} 17$ could differentially regulate MYB gene pairs' expression when the BR signaling was blocked (Supplementary Figure S2). Populus MYB gene pairs, such as $P d M Y B 90 / 167$ and $P d M Y B 92 / 125$, were identified to coordinately regulate secondary wall deposition, as well as affected by $\mathrm{PdC} 3 \mathrm{H} 17$ during xylem formation (Chai et al., 2014a,b). The different expression patterns in Supplementary Figure S2 indicated that BR might be associated with different pathways to modulate these $\mathrm{C} 3 \mathrm{H} 17$ mediated gene pairs. Additionally, the xylem formation in PdC3H17OE showed less sensitivity to BR in our study (Figure 4), probably because the endogenous transcript of $\mathrm{PdC} 3 \mathrm{H} 17$ induced by BR treatment in $P d C 3 H 17 O E$ was not as much as in the control and PdC3H17DR due to feedback inhibition. On the contrary, the PCZ treatment had the opposite effect as 


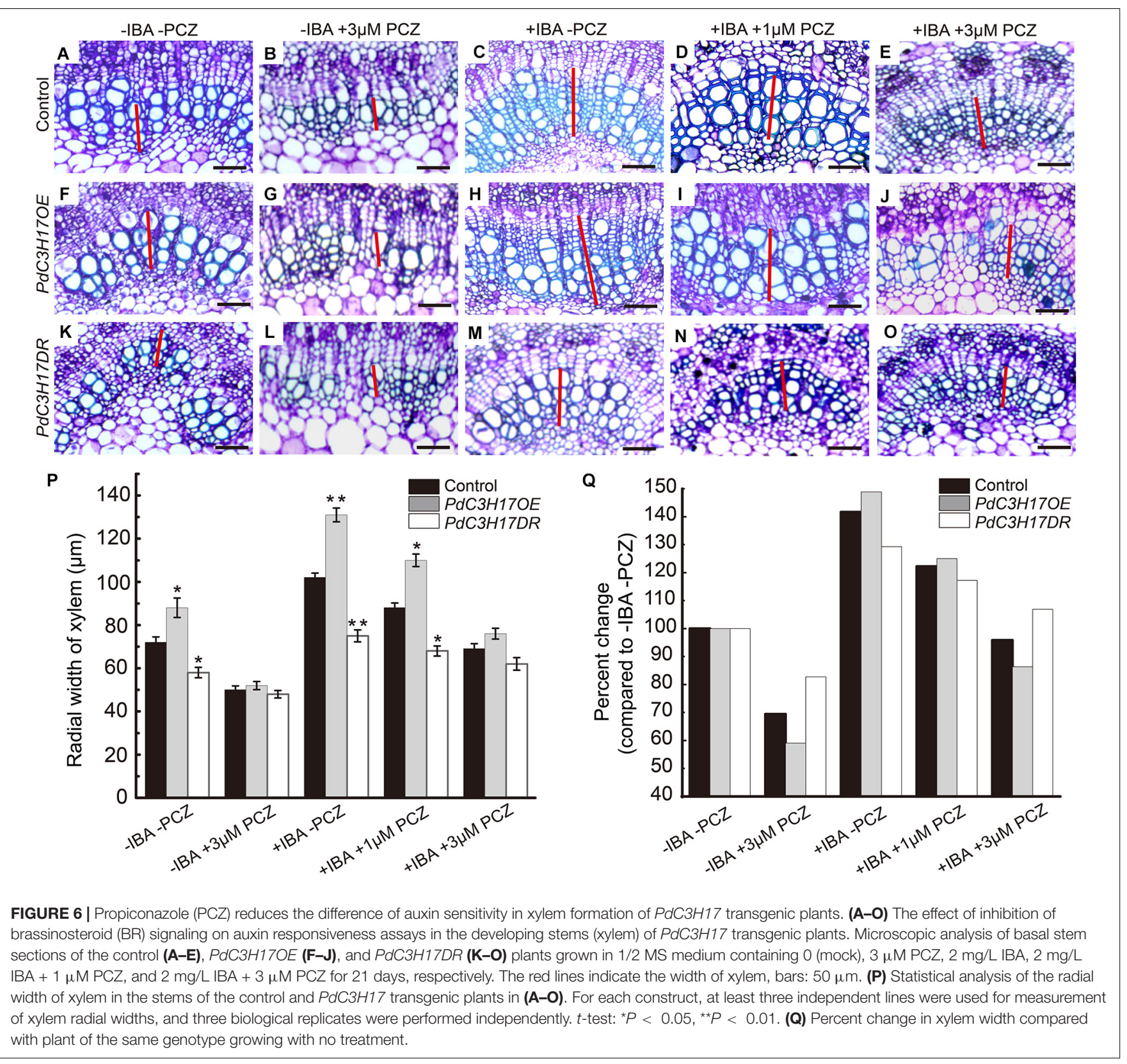

compared with $\mathrm{BL}$ in $\mathrm{PdC} 3 \mathrm{H} 17 \mathrm{OE}$, and the xylem formation in PdC3H17OE was drastically suppressed compared with control (Figure 3). One possible explanation for the results may be that $\mathrm{BR}$ has a posttranscriptional regulation on $\mathrm{PdC} 3 \mathrm{H} 17$ besides the transcriptional regulation, and the functional $\mathrm{PdC} 3 \mathrm{H} 17$ may require appropriate $\mathrm{BR}$ level. This type of regulation was previously reported. For instance, the $\mathrm{C} 3 \mathrm{H}$-type transcription factor LIC antagonizes BZR1 to repress BR signaling in rice. LIC transcription was rapidly induced by BR treatment and was found to function as a dephosphorylation form at high BR level (Zhang et al., 2012).

As a key regulator in both BR and TIDIIF-GSKs signaling pathways, BES1 has an important role in promoting xylem formation in Arabidopsis (Saito et al., 2018), but its downstream regulation network is still unclear. $\mathrm{PdC} 3 \mathrm{H} 17$ was confirmed to be a target of $\mathrm{PdMYB} / 21$, secondary top regulator in poplar xylem formation (Chai et al., 2014a). In the current study, we showed that $P d C 3 H 17$ could also be a target of PdBES1 in the BR signaling during xylem formation. The in situ assay revealed that $P d C 3 H 17$ and PdBES1 had a highly overlapped expression profile within the wood-forming tissues, indicating a direct connection between these two genes. The subsequent molecular analyses including $\mathrm{Y} 1 \mathrm{H}$, transcription activation assays, and EMSA analysis further confirmed that $\mathrm{PdC} 3 \mathrm{H} 17$ was involved in BR signaling through the direct regulation by PdBES1 (Figure 1). Although the precise role of poplar PdBES1 in xylem formation has not been explored, the evidences in our study indicated that PdBES1 probably functioned in poplar xylem formation. For 


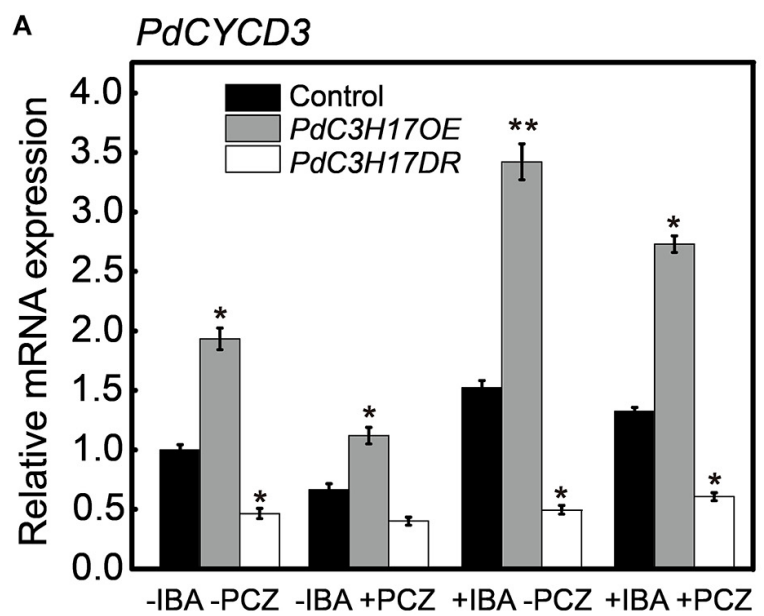

C

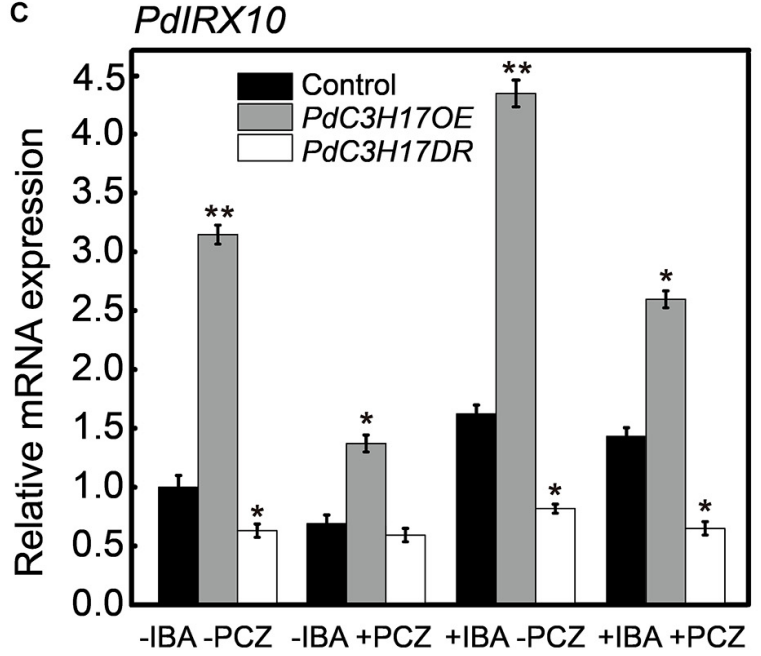

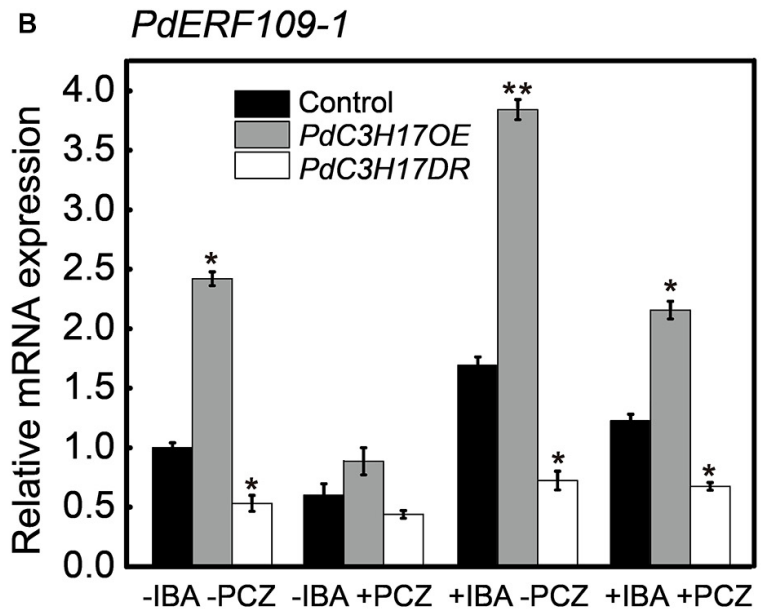

D PdIRX15L-1

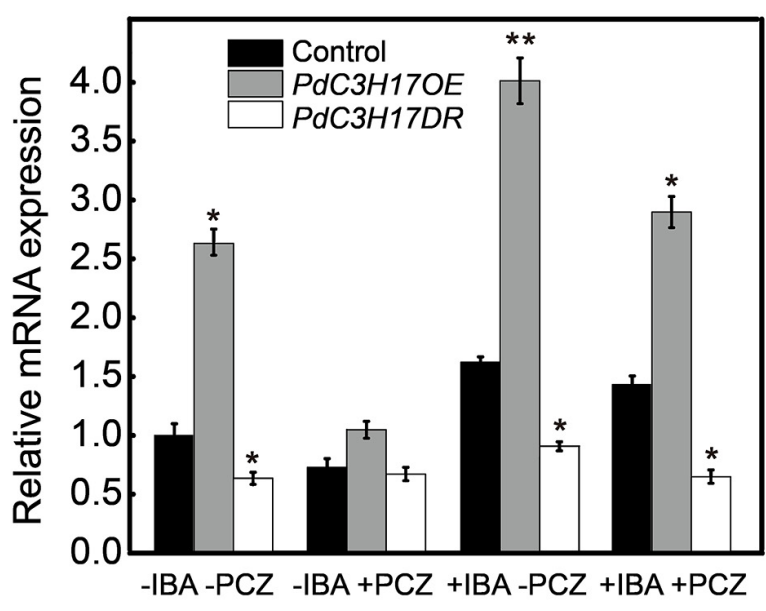

FIGURE 7 | Brassinosteroid (BR) influences the transcription of auxin promotion of genes in PdC3H17 transgenic plants. (A-D) qRT-PCR analysis of two auxin-mediated cell division related genes of PdCYCD3 (A) and PdERF109-1 (B), two secondary cell wall deposition-related genes of PdIRX10 (C), and PdIRX15L-1 (D) in PdC3H17 transgenic plants treated with 0, $2 \mathrm{mg} / \mathrm{L}$ IBA, and $2 \mathrm{mg} / \mathrm{L}$ IBA $+3 \mu \mathrm{M}$ propiconazole (PCZ) for $8 \mathrm{~h}$, respectively. Data are means \pm SD of three biological repeats. $t$-test: ${ }^{\star} P<0.05,{ }^{\star \star} P<0.01$.

instance, PdBES1 shared more than $70 \%$ amino acid similarity with AtBES1, implying these two homologous proteins might be functionally conserved in xylem formation. Additionally, PdBES1 had a higher transcript level in the stem in tissue expression analysis and was predominantly distributed in the cambium and developing xylem section according to in situ and AspWood RNAseq assays (Figure 2), revealing that PdBES1 has a potential role in xylem formation.

The crosstalk of BRs and auxin plays crucial roles in many aspects of plant growth and development, such as cell elongation, organ pattern formation, and vascular development (Woodward and Bartel, 2005; Altmann et al., 2020). For instance, SMOS1 and SMOS2/DLT integrate auxin and BR signalings to regulate lamina joint bending in rice (Hirano et al., 2017). Favero et al. (2017) revealed that SOB3 could converge with BR and auxin signalings to influence hypocotyl growth. In addition, vascularrelated unknown protein 1 (VUP1) was considered to regulate secondary wall formation during vascular development through modulating the expression of many BR- and auxin-responsive genes in Arabidopsis (Grienenberger and Douglas, 2014). In the current study, we showed a novel type of crosstalk between auxin and BR signaling occurred through PdC3H17 during xylem formation. Regulation of PdC3H17-mediated xylem formation by auxin signaling was previously reported by Tang et al. (2020) and further confirmed in this study. Moreover, the inhibition of BR signaling could obviously reduce the sensitivity of $\mathrm{PdC} 3 \mathrm{H} 17$ transgenic plants in auxin-mediated xylem formation (Figure 6). During the co-regulated biological processes, BR and auxin signaling shares many transcriptional target genes. For example, the SMOS1-SMOS2 complex regulates joint bending through the regulation of $O S P H I-1$, and $S O B 3$ modulates hypocotyl elongation mediated by auxin and BR through influencing SAUR19 subfamily transcription (Favero et al., 2017; Hirano et al., 2017). In our results, the expression of some xylem-related genes 
affected by auxin in previous study was also changed by PCZ treatment, indicating these genes were common targets for the two hormones and were responsible for the alternation of xylem formation observed in $\mathrm{PdC} 3 \mathrm{H} 17$ transgenic plants (Figure 7).

Previous reports suggested that interactions between TFs involved in auxin and BR signaling might act as the points of auxin-BR crosstalk; one case is the response of SAUR15 to BR and auxin. The expression of SAUR15 depends on the combined binding of BES1 and MONOPTEROS/ARF5 within its promoter harboring a hormone up at dawn-type E-box and AuxRE cis elements, respectively (Walcher and Nemhauser, 2012). In our study, we also found that there were auxin response elements within promoter of $\mathrm{PdC} 3 \mathrm{H} 17$ (data not shown), which inferred that PdC3H17 could combine these two hormones through BES1 and other ARFs during xylem formation. Future studies should investigate which ARFs are involved in this interaction.

\section{CONCLUSION}

Our study revealed $\mathrm{PdC} 3 \mathrm{H} 17$ was involved in BR signaling during xylem formation and might be a target of PdBES1 at the transcriptional level. Moreover, $\mathrm{PdC} 3 \mathrm{H} 17$ could also be a crosstalk point of the auxin and BR signaling pathways in this process. However, how these hormones act together to regulate vascular development need far more explored. Our findings provide new insights into the mechanism of BRs on xylem formation and the interaction with other hormones within this process.

\section{DATA AVAILABILITY STATEMENT}

Publicly available datasets were analyzed in this study. This data can be found here: AspWood, http://aspwood.popgenie.org.

\section{REFERENCES}

Altmann, M., Altmann, S., Rodriguez, P. A., Weller, B., Vergara, L. E., Palme, J., et al. (2020). Extensive signal integration by the phytohormone protein network. Nature 583, 271-276. doi: 10.1038/s41586-020-2460-0

Bao, F., Shen, J., Brady, S. R., Muday, G. K., Asami, T., and Yang, Z. (2004). Brassinosteroids interact with auxin to promote lateral root development in Arabidopsis. Plant Physiol. 34, 1624-1631. doi: 10.1104/pp.103.036897

Bhalerao, R. P., and Fischer, U. (2014). Auxin gradients across wood-instructive or incidental? Physiol Plantarum. 151, 43-51. doi: 10.1111/ppl.12134

Cano-Delgado, A., Yin, Y., Yu, C., Vafeados, D., Mora-Garcla, S., Cheng, J. C., et al. (2004). BRL1 and BRL3 are novel brassinosteroid receptors that function in vascular differentiation in Arabidopsis. Development. 131, 5341-5351. doi: 10.1242/dev.01403

Chai, G. H., Qi, G., Yu, L., Tang, X., Yu, Y. C., Wang, D., et al. (2014a). Poplar PdC3H17 and PdC3H18 are direct targets of PdMYB3 and PdMYB21 and positively regulate secondary wall formation in Arabidopsis and poplar. New Phytol. 203, 520-534. doi: 10.1111/nph.12825

Chai, G. H., Wang, Z., Tang, X. F., Yu, L., Qi, G., Wang, D., et al. (2014b). R2R3MYB gene pairs in Populus: evolution and contribution to secondary wall formation and flowering. J. Exp. Bot. 65, 4255-4242. doi: 10.1093/jxb/eru196

Du, J., Gerttula, S., Li, Z. H., Zhao, S. T., Li, L. Y., Liu, Y., et al. (2020). Brassinosteroid regulation of wood formation in poplar. New Phytol. 225, 1516-1530. doi: 10.1111/nph.15936

\section{AUTHOR CONTRIBUTIONS}

XT, SL, and GZ designed research. XT, CW, YL, NM, and $\mathrm{GH}$ conducted the experiments and analyzed the data. XT and HX wrote the manuscript. SL, GC, HX, and GZ revised the manuscript. All authors read and approved the manuscript.

\section{FUNDING}

This work was supported by the National Key Program on Transgenic Research (2018ZX08020002), the National Key Scientific Research Project of China (2016YFD0600104), National Natural Science Foundation of China (31701068, 31570670, and 31770315), Shandong Provincial Natural Science Foundation (ZR2017BC096), Major Basic Research Project of Shandong Natural Science Foundation (ZR2018ZC0335), the First Class Grassland Science Discipline Program of Shandong Province, and the Taishan Scholar Program of Shandong Province (to GZ).

\section{SUPPLEMENTARY MATERIAL}

The Supplementary Material for this article can be found online at: https://www.frontiersin.org/articles/10.3389/fpls.2020. 586014/full\#supplementary-material

Supplementary Figure 1 | Diagram summarizing of cis-elements in the promoter region upstream of start code of $\mathrm{PdC} 3 \mathrm{H} 17$.

Supplementary Figure $\mathbf{2} \mid$ Brassinosteroid (BR) affects the expression of a subset of $\mathrm{PdC} 3 \mathrm{H} 17$-mediated MYB transcription factors.

Supplementary Table 1 | Primers used in this study.

Supplementary Table 2 | List of putative candidate transcription factors regulating $\mathrm{PdC} 3 \mathrm{H} 17$ expression identified by yeast one-hybrid assay.

Fan, C. F., Yu, H., Qin, S. F., Li, Y. L., Alam, A., Xu, C. Z., et al. (2020). Brassinosteroid Overproduction improves lignocellulose quantity and quality to maximize bioethanol yield under green-like biomass process in transgenic poplar. Biotechnol. Biofuels. 13:9. doi: 10.1186/s13068-020-1652-z

Favero, D. S., Le, K. N., and Neff, M. M. (2017). Brassinosteroid signaling converges with suppressor of phytochrome B4-\#3 to influence the expression of small auxin up RNA genes and hypocotyl growth. Plant J. 89, 1133-1145. doi: 10. $1111 /$ tpj.13451

Fu, M., and Blackshear, P. J. (2017). RNA-binding proteins in immune regulation: a focus on CCCH zinc finger proteins. Nat. Rev. Immunol. 17, 130-143. doi: 10.1038/nri.2016.129

Grienenberger, E., and Douglas, C. J. (2014). Arabidopsis vascular-related unknown protein1 regulates Xylem development and growth by a conserved mechanism that modulates hormone signaling. Plant Physiol. 164, 1991-2010. doi: 10.1104/ pp.114.236406

Guo, H., Li, L., Aluru, M., and Yin, Y. (2013). Mechanisms and networks for brassinosteroid regulated gene expression. Curr. Opin. Plant Biol. 16, 545-553. doi: 10.1016/j.pbi.2013.08.002

Hardtke, C. S. (2007). Transcriptional auxin-brassinosteroid crosstalk: who's talking? Bioessays 29, 1115-1123. doi: 10.1002/bies.20653

He, J. X., Gendron, J. M., Sun, Y., Gampala, S. S., Gendron, N., Sun, C. Q., et al. (2005). BZR1 is a transcriptional repressor with dual roles in brassinosteroid homeostasis and growth responses. Science 307, 1634-1638. doi: 10.1126/ science. 1107580 
Hirano, K., Yoshida, H., Aya, K., Kawamura, M., Hayashi, M., Hobo, T., et al. (2017). Small organ sizel and small organ size 2/DWARF and low-tillering form a complex to integrate auxin and brassinosteroid signaling in rice. Mol Plant. 10, 590-604. doi: 10.1016/j.molp.2016.12.013

Hudson, B. P., Martinez-Yamout, M. A., Dyson, H. J., and Wright, P. E. (2004). Recognition of the mRNA AU-rich Element by the zinc finger domain of TIS11d. Nat. Struct. Mol. Biol. 11, 257-264. doi: 10.1038/ nsmb738

Ibanes, M., Fabregas, N., Chory, J., and Cano-Delgado, A. I. (2009). Brassinosteroid signaling and auxin transport are required to establish the periodic pattern of Arabidopsis shoot vascular bundles. Proc. Natl. Acad. Sci. U.S.A. 106, 1363013635. doi: 10.1073/pnas.0906416106

Immanen, J., Nieminen, K., Smolander, O. P., Kojima, M., Alonso, S. J., Koskinen, P., et al. (2016). Cytokinin and auxin display distinct but interconnected distribution and signaling profiles to stimulate cambial activity. Curr. Biol. 26, 1990-1997. doi: 10.1016/j.cub.2016.05.053

Jin, H., Do, J., Shin, S. J., Choi, J. W., Choi, Y. I., Kim, W., et al. (2014). Exogenously applied 24-epi brassinolide reduces lignification and alters cell wall carbohydrate biosynthesis in the secondary xylem of Liriodendron tulipifera. Phytochemistry 101, 40-51. doi: 10.1016/j.phytochem.2014.02.003

Jin, Y., Tang, R., Wang, H., Jiang, C., Bao, Y., Yang, Y., et al. (2017). Overexpression of populus trichocarpa CYP85A3 promotes growth and biomass production in transgenic trees. Plant Biotechnol. J. 15, 1309-1321. doi: 10.1111/pbi.12717

Jin, Y. L., Yu, C. Y., Jiang, C. M., Guo, X. T., Li, B., Wang, C. T., et al. (2020). PtiCYP85A3, a BR C-6 oxidase gene, plays a critical role in brassinosteroidmediated tension wood formation in poplar. Front Plant Sci. 11:468. doi: 10. 3389/fpls.2020.00468

Jouannet, V., Brackmann, K., and Greb, T. (2015). (Pro)cambium formation and proliferation: two sides of the same coin? Curr. Opin. Plant Biol. 23, 54-60. doi: 10.1016/j.pbi.2014.10.010

Kang, Y. H., Breda, A., and Hardtke, C. S. (2017). Brassinosteroid signaling directs formative cell divisions and protophloem differentiation in Arabidopsis root meristems. Development 144, 272-280. doi: 10.1242/dev.145623

Kim, D. H., Yamaguchi, S., Lim, S., Oh, E., Park, J., Hanada, A., et al. (2008). SOMNUS, a CCCH-type zinc finger protein in Arabidopsis, negatively regulates light-dependent seed germination downstream of PIL5. Plant Cell 20, 12601277. doi: 10.1105/tpc.108.058859

Kondo, Y., Fujita, T., Sugiyama, M., and Fukuda, H. (2015). A novel system for xylem cell diferentiation in Arabidopsis thaliana. Mol. Plant. 8, 612-621. doi: 10.1016/j.molp.2014.10.008

Kondo, Y., Ito, T., Nakagami, H., Hirakawa, Y., Saito, M., Tamaki, T., et al. (2014). Plant GSK3 proteins regulate xylem cell differentiation downstream of TDIF-TDR signalling. Nat. Commun. 5:3504. doi: 10.1038/ncomms4504

Lai, W. S., and Blackshear, P. J. (2001). Interactions of $\mathrm{CCCH}$ zinc finger proteins with mRNA: tristetraprolin-mediated AU-rich element-dependent mRNA degradation can occur in the absence of a poly(A) tail. J. Biol. Chem. 276, 23144-23154. doi: 10.1074/jbc.M100680200

Lai, W. S., Carballo, E., Thorn, J. M., Kennington, E. A., and Blackshear, P. J. (2000). Interactions of $\mathrm{CCCH}$ zinc finger proteins with mRNA. Binding of tristetraprolin-related zinc finger proteins to Au-rich elements and destabilization of mRNA. J. Biol. Chem. 275, 17827-17837. doi: 10.1074/jbc. M110395200

Li, C., Ma, X., Yu, H., Fu, Y., and Luo, K. (2018). Ectopic expression of Ptomyb74 in poplar and arabidopsis promotes secondary cell wall formation. Front. Plant Sci. 9:1262. doi: 10.3389/fpls.2018.01262

Noh, S. A., Choi, Y. I., Cho, J. S., and Lee, H. (2015). The poplar basic helixloop helix transcription factor BEE3-Like gene affects biomass production by enhancing proliferation of xylem cells in poplar. Biochem. Biophys. Res. Commun. 462, 64-70. doi: 10.1016/j.bbrc.2015.04.109

Oh, E., Zhu, J. Y., Bai, M. Y., Arenhart, R. A., Sun, Y., and Wang, Z. Y. (2014). Cell elongation is regulated through a central circuit of interacting transcription factors in the Arabidopsis hypocotyl. elife 3:e03031. doi: 10.7554/eLife.03031.001

Ragni, L., Nieminen, K., Pacheco-Villalobos, D., Sibout, R., Schwechheimer, C., and Hardtke, C. S. (2011). Mobile gibberellin directly stimulates Arabidopsis hypocotyl xylem expansion. Plant Cell. 23, 1322-1336. doi: 10.1105/tpc.111. 084020
Reinhardt, D., Pesce, E. R., Stieger, P., Mandel, T., Baltensperger, K., Bennett, M., et al. (2003). Regulation of phyllotaxis by polar auxin transport. Nature 426, 255-260. doi: 10.1038/nature02081

Ruzicka, K., Ursache, R., Hejatko, J., and Helariutta, Y. (2015). Xylem development from the cradle to the grave. New Phytol. 207, 519-535. doi: 10.1111/nph.13383

Ryu, H., Cho, H., Kim, K., and Hwang, I. (2010). Phosphorylation dependent nucleocytoplasmic shuttling of BES1 is a key regulatory event in brassinosteroid signaling. Mol. Cells 29, 283-290. doi: 10.1007/s10059-0100035-X

Saito, M., Kondo, Y., and Fukuda, H. (2018). BES1 and BZR1 redundantly promote phloem and xylem differentiation. Plant Cell Physiol. 59, 590-600. doi: 10.1093/ pcp/pcy012

Sauer, M., Balla, J., Luschnig, C., Wisniewska, J., Reinohl, V., Friml, J., et al. (2006). Canalization of auxin flow by Aux/IAA-ARF-dependent feedback regulation of PIN polarity. Genes Dev. 20, 2902-2911. doi: 10.1101/gad.390806

Schaller, G. E., Bishopp, A., and Kieber, J. J. (2015). The yin-yang of hormones: cytokinin and auxin interactions in plant development. Plant Cell 27, 44-63. doi: $10.1105 /$ tpc.114.133595

Seok, H. Y., Woo, D. H., Park, H. Y., Lee, S. Y., Tran, H. T., Lee, E. H., et al. (2016). AtC3H17, a non-tandem $\mathrm{CCCH}$ zinc finger protein, functions as a nuclear transcriptional activator and has pleiotropic effects on vegetative development, flowering and seed development in Arabidopsis. Plant Cell Physiol. 57, 603-615. doi: 10.1093/pcp/pcw013

Shen, Y., Li, Y. L., Xu, D., Yang, C., Li, C. F., and Luo, K. M. (2018). Molecular cloning and characterization of a brassinosteroid biosynthesis-related gene PtoDWF4 from Populus tomentosa. Tree Physiol. 38, 1424-1436. doi: 10.1093/ treephys/tpy027

Sorce, C., Giovannelli, A., Sebastiani, L., and Anfodillo, T. (2013). Hormonal signals involved in the regulation of cambial activity, xylogenesis and vessel patterning in trees. Plant Cell. Rep. 32, 885-898. doi: 10.1007/s00299-013-14314

Sundell, D., Street, N. R., Kumar, M., Mellerowicz, E. J., Kucukoglu, M., Johnsson, C., et al. (2017). AspWood: high-spatial-resolution transcriptome profifiles reveal uncharacterized modularity of wood formation in Populus tremula. Plant Cell 29, 1585-1604. doi: 10.1105/tpc.17.00153

Tang, X. F., Wang, D., Liu, Y., Lu, M. Z., Zhuang, Y. M., Xie, Z., et al. (2020). Dual regulation of xylem formation by an auxin-mediated PaC3H17PaMYB199 module in Populus. New Phytol. 225, 1545-1561. doi: 10.1111/nph. 16244

Tang, X. F., Zhuang, Y. M., Qi, G., Wang, D., Liu, H., Wang, K., et al. (2015). Poplar PdMYB221 is involved in the direct and indirect regulation of secondary wall biosynthesis during wood formation. Sci. Rep. 16:12240. doi: 10.1038/ srep 12240

Walcher, C. L., and Nemhauser, J. L. (2012). Bipartite promoter element required for auxin response. Plant Physiol. 158, 273-282. doi: 10.1002/pld3.166

Wang, Y. P., Xu, Y., Pei, S. Q., Lu, M. M., Kong, Y. Z., Zhou, G. K., et al. (2020). KNAT7 modulates xylan biosynthesis in Arabidopsis seed coat mucilage. J. Exp. Bot. 20:eraa189. doi: 10.1093/jxb/eraa189

Woodward, A. W., and Bartel, B. (2005). Auxin: regulation, action, and interaction. Ann. Bot. 95, 707-735. doi: 10.3390/biom 10050746

Xu, C., Shen, Y., He, F., Fu, X., Yu, H., Lu, W., et al. (2019). Auxin-mediated Aux/IAA-ARF-HB signaling cascade regulates secondary xylem development in Populus. New Phytol. 222, 752-767. doi: 10.1111/nph.15658

Xu, Z., Song, N., Ma, L., and Wu, J. (2019). IRE1-bZIP60 pathway is required for nicotiana attenuata resistance to fungal pathogen alternaria alternata. Front. Plant Sci. 10:263. doi: 10.3389/fpls.2019.00263

Yamamoto, R., Demura, T., and Fukuda, H. (1997). Brassinosteroids induce entry into the final stage of tracheary element differentiation in cultured Zinnia cells. Plant Cell. Physio. 38, 980-983. doi: 10.1093/oxfordjournals.pcp.a029262

Yamamoto, R., Fujioka, S., Demura, T., Takatsuto, S., Yoshida, S., and Fukuda, H. (2001). Brassinosteroid levels increase drastically prior to morphogenesis of tracheary elements. Plant Physiol. 125, 556-563. doi: 10.1104/pp.125.2.556

Yin, Y. H., Wang, Z. Y., Mora-Garcia, S., Li, J. M., Yoshida, S., Asami, T., et al. (2002). BES1 accumulates in the nucleus in response to brassinosteroids to regulate gene expression and promote stem elongation. Cell 109, 181-191. doi: 10.1016/s0092-8674(02)00721-3 
Zhang, C., Xu, Y., Guo, S., Zhu, J., Huan, Q., Liu, H., et al. (2012). Dynamics of brassinosteroid response modulated by negative regulator LIC in rice. PLoS Genet. 8:e1002686. doi: 10.1371/journal.pgen.1002686

Zhang, H., Gao, X., Zhi, Y., Li, X., Zhang, Q., Niu, J., et al. (2019). A nontandem CCCH-type zinc-finger protein, IbC3H18, functions as a nuclear transcriptional activator and enhances abiotic stress tolerance in sweet potato. New Phytol. 223, 1918-1936. doi: 10.1111/nph.15925

Zhong, R., McCarthy, R. L., Lee, C., and Ye, Z. H. (2011). Dissection of the transcriptional program regulating secondary wall biosynthesis during wood formation in poplar. Plant Physiol. 157, 1452-1468. doi: 10.1104/pp.111. 181354
Conflict of Interest: The authors declare that the research was conducted in the absence of any commercial or financial relationships that could be construed as a potential conflict of interest.

Copyright (c) 2020 Tang, Wang, Liu, He, Ma, Chai, Li, Xu and Zhou. This is an open-access article distributed under the terms of the Creative Commons Attribution License (CC BY). The use, distribution or reproduction in other forums is permitted, provided the original author(s) and the copyright owner(s) are credited and that the original publication in this journal is cited, in accordance with accepted academic practice. No use, distribution or reproduction is permitted which does not comply with these terms. 